\title{
Successful management of prolonged venovenous extracorporeal membrane oxygenation in an octogenarian
}

\author{
Masami Takagaki $^{1} \cdot$ Hiroki Yamaguchi $^{1} \cdot$ Shinichi Mitsuyama $^{1} \cdot$ Tasuku Kadowaki $^{1}$. \\ Takeshi Ando 1
}

Received: 26 July 2017 / Accepted: 25 September 2017 / Published online: 6 October 2017

(C) The Japanese Society for Artificial Organs 2017

\begin{abstract}
Venovenous extracorporeal membrane oxygenation is now an established treatment for acute respiratory distress syndrome. However, this treatment remains rare in octogenarians and is associated with poor outcomes. An 81-year-old man with a history of chronic obstructive pulmonary disease and heavy smoking underwent mitral and tricuspid valve repair and the Maze procedure for mitral and tricuspid regurgitation and paroxysmal atrial fibrillation. Although he was extubated the following day, his postoperative course was complicated with pneumonia followed by acute respiratory distress syndrome. He was reintubated on day 7. Ratio of partial pressure of arterial oxygen to fraction of inspired oxygen continuously dropped to less than $100 \mathrm{mmHg}$, and venovenous extracorporeal membrane oxygenation support was induced on day 18 . His lung condition showed slow and steady recovery, and he was successfully weaned from mechanical support on day 44 (total support, 27 days). Bleeding complication from tracheotomy (day 31) due to disseminated intravascular coagulation was successfully managed using recombinant human soluble thrombomodulin. He was ambulatory and discharged to a nursing facility without tracheotomy on day 172 . Proper extracorporeal membrane oxygenation management, while challenging to keep the elderly patient away from further complications, saved an 81-year-old patient.
\end{abstract}

Masami Takagaki

takagam2004@yahoo.co.jp

Hiroki Yamaguchi

hiroyamaguchiskt@gmail.com

1 Department of Cardiovascular Surgery, Showa University Koto Toyosu Hospital, 5-1-38 Toyosu, Koto-ku, Tokyo 135-8577, Japan
Keywords Extracorporeal membrane oxygenation · Octogenarian · Acute respiratory distress syndrome . Prolonged respiratory failure

\section{Introduction}

Venovenous extracorporeal membrane oxygenation (ECMO) is now an established treatment for acute respiratory distress syndrome (ARDS) [1], which protects the lungs from stretch-induced lung injury, thus reducing mortality [2]. We have actively introduced this treatment to post-cardiotomy ARDS [3]. Recent substantial advances in ECMO technology have made longer-term applications feasible [4]. The largest series to date, conducted by Camboni and associates [5], showed no significant difference in the survival of patients requiring ECMO for more than 3 weeks. However, ECMO support in octogenarians remains rare and is associated with poor outcomes, with approximately $80 \%$ inhospital mortality [6]. We hereby report a case of an 81-year-old man who recovered from post-cardiotomy ARDS after successful management of ECMO support for 27 days.

\section{Case presentation}

The patient was an 81-year-old man who had a history of chronic obstructive lung disease and heavy smoking (4 packs/day for 37 years). He had three episodes of hospital admission due to congestive heart failure symptoms (New York Heart Association class III). Significant mitral and tricuspid regurgitations were identified by a local cardiologist as well as paroxysmal atrial fibrillation. He was then referred to us for surgery. His echocardiography showed left ventricular dilatation (end-diastolic diameter, $61 \mathrm{~mm}$ ) with 
depressed systolic function (ejection fraction, 38\%), severe functional mitral regurgitation with leaflet tethering, and moderate tricuspid regurgitation with annular dilatation. $\mathrm{He}$ was diagnosed as a candidate for mitral and tricuspid valve repair and the Maze procedure.

The patient underwent surgery uneventfully. The mitral valve was repaired by posterior leaflet augmentation using autologous pericardium and ring annuloplasty, and the tricuspid valve was repaired by annuloplasty only. The left atrial appendage was amputated, and the modified biatrial Maze procedure was conducted using a radiofrequency device and cryoablation. Aortic clamp time and cardiopulmonary bypass time were 168 and $214 \mathrm{~min}$, respectively. The surgery lasted $7 \mathrm{~h}$ and $20 \mathrm{~min}$.

The patient's postoperative course was initially smooth. Upon Intensive Care Unit (ICU) admission, his Acute Physiology and Chronic Health Evaluation III score was
60 , predicting the length of ICU stay to be approximately 6 days [7]. His Sequential Organ Failure Assessment (SOFA) score was 8 [8]. He was extubated the day after surgery with good oxygenation. Partial pressure of arterial oxygen $\left(\mathrm{PaO}_{2}\right)$ to fraction of inspired oxygen $\left(\mathrm{FiO}_{2}\right)$ ratio ( $P / F$ ratio) was $392 \mathrm{mmHg}$ (Fig. 1). Chest X-ray showed no infiltration (Fig. 2a). However, he showed worsening oxygenation on day 2 , requiring noninvasive positive pressure ventilation. He had a spike fever on day 6 and was diagnosed with pneumonia. He was reintubated on day 7. Administration of inhaled nitric oxide was started on day 8 to minimize ventilation-perfusion mismatching. Methicillin-resistant staphylococcus aureus was detected from sputum culture, and vancomycin was started on day 8 . Steroid pulse therapy was conducted on days $8-10$. Although his $P / F$ ratio temporarily recovered from less than $100 \mathrm{mmHg}$ to approximately $200 \mathrm{mmHg}$ (Fig. 1), his condition once again deteriorated,
Fig. 1 Trend in the ratio of partial pressure of arterial oxygen to fraction of inspired oxygen during ECMO support. Although the $P / F$ ratio worsened after ECMO induction, it began to improve after tracheotomy with active physiotherapy and steroid therapy under a lung-protective protocol. ECMO, Extracorporeal membrane oxygenation; $\mathrm{FiO}_{2}$, fraction of inspired oxygen; $\mathrm{PaO}_{2}$, partial pressure of arterial oxygen; $P / F$ ratio, ratio of partial pressure of arterial oxygen to fraction of inspired oxygen

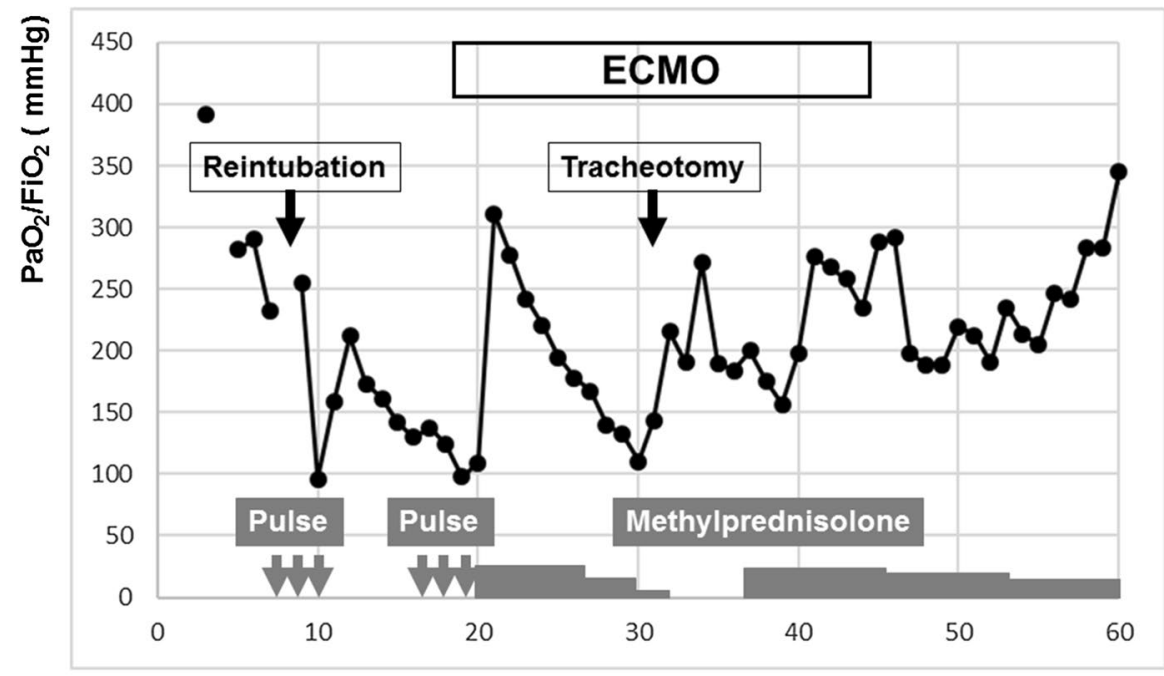

Postoperative Days
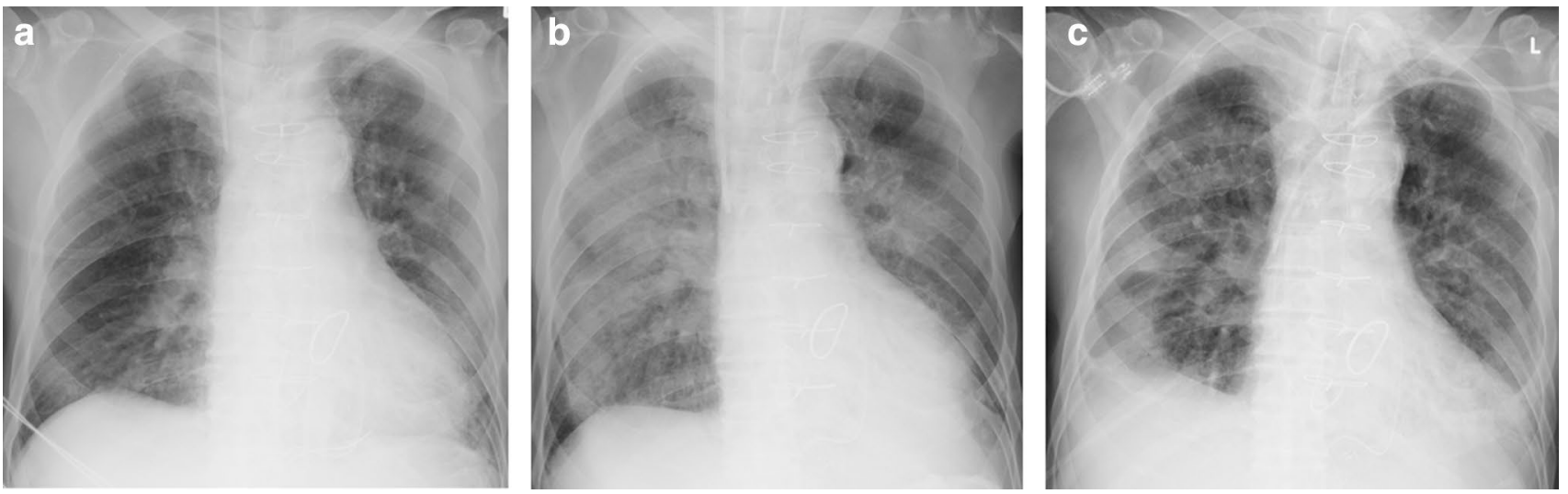

Fig. 2 Chest X-ray before and after the ECMO support. a Chest $\mathrm{X}$-ray just after extubation shows no infiltration. b Chest X-ray before the induction of venovenous ECMO shows bilateral lung infiltration. c Chest X-ray shows improvement after prolonged ECMO support. ECMO, Extracorporeal membrane oxygenation 
showing appearance of foamy expectoration and typical ARDS shadow on chest X-ray (Fig. 2b) with worsening ventilator support $\left[\mathrm{FiO}_{2}=0.8\right.$, positive end-expiratory pressure $(\mathrm{PEEP})=10 \mathrm{~cm} \mathrm{H}_{2} \mathrm{O}$, peak airway pressure (PIP) $=26 \mathrm{~mm}$ $\mathrm{H}_{2} \mathrm{O}$ ]. Second steroid pulse between days 17-19 was conducted without improvement. On day 18, his SOFA score increased to 12 simply due to worsening $P / F$ to less than $100 \mathrm{~mm} \mathrm{Hg}\left(\mathrm{pH}, 7.445 ; \mathrm{PaO}_{2}, 68.3 \mathrm{~mm} \mathrm{Hg} ; \mathrm{PaCO}_{2}\right.$, $40.0 \mathrm{~mm} \mathrm{Hg}$; $\mathrm{FiO}_{2}$, 1.0; PEEP, $10 \mathrm{~cm} \mathrm{H}_{2} \mathrm{O}$; and PIP, $26 \mathrm{~mm}$ $\mathrm{H}_{2} \mathrm{O}$ ). The patient was febrile (temperature, $38.3{ }^{\circ} \mathrm{C}$ ) with an elevated white blood cell count of $18440 / \mu 1$ and C-reactive protein level of $11.50 \mathrm{mg} / \mathrm{dl}$. However, blood cultures were all negative, and the procalcitonin level was not very high at $0.15 \mathrm{ng} / \mathrm{ml}$. Most importantly, the patient showed no other signs of organ dysfunction. The patient was sedated by propofol without any abnormal neurological signs. His mean arterial pressure was stable at $81 \mathrm{mmHg}$ with low-dose epinephrine and norepinephrine, and his platelet count was $29.8 \times 10^{4} / \mu 1$. Additionally, his total bilirubin level decreased from $3.6 \mathrm{mg} / \mathrm{dl}$ after reintubation to $2.3 \mathrm{mg} / \mathrm{dl}$, and his creatinine level improved from $2.12 \mathrm{mg} / \mathrm{dl}$ just after surgery to $1.01 \mathrm{mg} / \mathrm{dl}$, with urine output of more than $3000 \mathrm{ml} /$ day. We decided to introduce a venovenous ECMO system, expecting that lung recovery can save this patient and prevent multiple organ failure (MOF).

The right femoral and right internal jugular veins were cannulated with a 20 Fr and a 16 Fr cannula, respectively, using the Seldinger technique. We used PCKC Cannulae with NSH heparin coating (SENKO MEDICAL INSTRUMENT Mfg. CO., LTD., Tokyo, Japan). Extracorporeal membrane oxygenation was initially induced using a MERA Centrifugal Pump NSH-R and a membrane oxygenator MERA NHP Excelung NSH-R (SENKO MEDICAL INSTRUMENT Mfg. CO., LTD.). Because we had to exchange the circuit twice due to plasma leakage from the artificial lung in the first 7 days, the $\mathrm{X}$ coating (TERUMO's polymer surface coating) system using a CAPIOX SL Centrifugal Pump and a CAPIOX LX Oxygenator (Terumo Corp., Tokyo Japan) was introduced on day 25 , with prolonged support expected.

Pump flow of $4.0 \mathrm{~L}$ min was initially achieved, and gradually decreased to $3.0 \mathrm{~L}$ min. The patient's $P / F$ ratio quickly improved to $300 \mathrm{mmHg}$ after ECMO induction (Fig. 1). The ventilator was then set to a lung-protective strategy with $\mathrm{FiO}_{2}$ of 0.40 , PEEP of $5 \mathrm{~cm} \mathrm{H}_{2} \mathrm{O}$, and PIP of $20 \mathrm{~cm} \mathrm{H}_{2} \mathrm{O}$. Anticoagulation was not used.

After ECMO induction, the patient's lung conditions worsened gradually, mainly related to ineffective sputum suction (physiotherapy) even utilizing prone position. Lowdose methylprednisolone $(0.5 \mathrm{mg} / \mathrm{kg})$ was started on day 20. Conventional tracheotomy was performed on day 31 . Although his lung condition improved with effective sputum suction after tracheotomy (Fig. 1), bleeding around the tracheotomy site was complicated without apparent surgical bleeding site. He was diagnosed with disseminated intravascular coagulation (DIC) with the Japanese association for acute medicine DIC score of 4 (decreased platelet count of $7.9 \times 10^{4} / \mu \mathrm{L}$ despite platelet transfusion and elevated fibrin degradation products of $78 \mu \mathrm{g} / \mathrm{ml}$ ). The cause of DIC was thought to be the ECMO support itself. We then proceeded to wean the patient off ECMO while managing the DIC using recombinant human soluble thrombomodulin (Recomodulin ${ }^{\circledR}$; Asahi Kasei Pharma Corporation Ltd., Tokyo, Japan), which has been officially approved for DIC in Japan since July 2015 and reported effective with less bleeding complications [9]. The FDP quickly decreased to $12 \mu \mathrm{g} / \mathrm{ml}$, without progression of bleeding.

ECMO support was successfully terminated on day 44 with improvement in chest X-ray (Fig. 2c). Steroid dose was gradually decreased as well. Bleeding from the tracheotomy site disappeared spontaneously after ECMO termination, and was accompanied by quick platelet count recovery. Signs of infection disappeared, and vancomycin was discontinued on day 55. Inhaled nitric oxide was discontinued on day 56. The patient's condition showed slow and steady recovery after ECMO termination, and he was transferred to the general ward on day 65 . He was weaned from the ventilator on day 115 , and tracheotomy tube was removed on day 141 . He was ambulatory and discharged to a nursing facility without tracheotomy on day 172 .

\section{Discussion and conclusions}

Recent advances in ECMO technology allow for longerterm ECMO management [4]. To date, several studies have been reported. The longest reported case was 193 days in a 30-year-old man with ARDS who was discharged with tracheotomy and was waiting for lung transplantation at the time of publication [10]. Patients in previous reports were very young, with the oldest case being approximately 50 years old [11]. Despite the progress of ECMO support technology, ECMO support for octogenarians remains rare and is associated with poor outcomes, with approximately $80 \%$ inhospital mortality [6]. Because we cannot expect lung transplant in elderly patients, a bridge-to-recovery protocol is the only option. Proper timing of ECMO induction, selection of patients, utilization of a lung protection protocol that prevents stretch injury, and management of complications such as bleeding are all keys to success for long-term ECMO support in octogenarians.

Our venovenous ECMO induction criteria are as follows: $\mathrm{PaO}_{2}$ less than $80 \mathrm{mmHg}$ and exacerbation of oxygenation or the appearance of foamy expectoration under maximum mechanical ventilation $\left(\mathrm{FiO}_{2}, 1.0\right.$; PEEP, $\left.10 \mathrm{mmHg}\right)$ [3]. We realized that advanced age has been traditionally considered 
as a relative contraindication for ECMO [12]. However, our octogenarians are preoperatively considered as candidates for cardiac surgery despite their age. We apply ECMO for acute lung damage during the postoperative period without such a relative contraindication. However, we agree that MOF is a contraindication for ECMO support. We need to induce ECMO early (prior to MOF) and prevent the development of MOF by proper management of complications.

Steroid therapy for ARDS is still controversial. Although there is no evidence to suggest that a short course of high dose steroids is helpful for either prevention or ARDS treatment [13], use of 3-day steroid pulse therapy has been demonstrated empirically for life-threatening ARDS, with expected beneficial effects on oxygenation in a declining clinical course [14]. In addition, prolonged treatment with low-dose corticosteroids for unresolving ARDS may be effective [13]. In this case, initial steroid pulse therapy at the time of reintubation seemed to be clinically effective, particularly for improvement of oxygenation as shown in Fig. 1. Although we cannot conclude anything regarding the effectiveness of steroid therapy in the present case, we will continue to utilize steroid therapy for ARDS, expecting improvement of oxygenation.

To conclude, management of prolonged ECMO in an octogenarian was challenging. However, proper ECMO management for 27 days and the prevention of MOF saved an 81-year-old patient.

Acknowledgements The authors would like to thank Enago (http:// www.enago.jp) for the English language review.

\section{Compliance with ethical standards}

Conflict of interest The authors declare that they have no conflicts of interest.

\section{References}

1. Brodie D, Bacchetta M. Extracorporeal membrane oxygenation for ARDS in adults. N Engl J Med. 2011;365:1905-14.

2. The Acute Respiratory Distress Syndrome Network, Brower RG, Matthay MA, Morris A, Schoenfeld D, Thompson BT, Wheeler A. Ventilation with lower tidal volumes as compared with traditional tidal volumes for acute lung injury and the acute respiratory distress syndrome. N Engl J Med 2000;342:1301-8.
3. Nakamura H, Yamaguchi H, Amano A, Nakao T. Venovenous extracorporeal membrane oxygenation is effective against postcardiotomy acute respiratory failure in adults. Gen Thorac Cardiovasc Surg. 2013;61:402-8.

4. Kon ZN, Dahi S, Evans CF, Byrnes KA, Bittle GJ, Wehman B, Rector RP, McCormick BM, Herr DL, Sanchez PG, Pham SM, Griffith BP. Long-term venovenous extracorporeal membrane oxygenation support for acute respiratory distress syndrome. Ann Thorac Surg. 2015;100:2059-63.

5. Camboni D, Philipp A, Lubnow M, Bein T, Haneya A, Diez C, Schmid C, Muller T. Support time-dependent outcome analysis for veno-venous extracorporeal membrane oxygenation. Eur J Cardiothorac Surg. 2011;40:1346-7.

6. Karagiannidis C, Brodie D, Strassmann S, Stoelben E, Philipp A, Bein T, Müller T, Windisch W. Extracorporeal membrane oxygenation: evolving epidemiology and mortality. Intensive Care Med. 2016;42:889-96.

7. Knaus WA, Wagner DP, Zimmerman JE, Draper EA. Variations in mortality and length of stay in intensive care units. Ann Intern Med. 1993;118:753-61.

8. Vincent JL, de Mendonça A, Cantraine F, Moreno R, Takala J, Suter PM, Sprung CL, Colardyn F, Blecher S, Ko WJ, Hsu HH, Tsai PR. Use of the SOFA score to assess the incidence of organ dysfunction/failure in intensive care units: results of a multicenter, prospective study. Working group on "sepsis-related problems" of the European Society of Intensive Care Medicine. Crit Care Med. 1998;26:1793-800.

9. Koami H, Sakamoto Y, Sakurai R, Ohta M, Imahase H, Yahata M, Umeka M, Miike T, Nagashima F, Iwamura T, Yamada KC, Inoue $\mathrm{S}$. The efficacy and associated bleeding complications of recombinant antithrombin supplementation among intensive care unit patients. Thromb Res. 2017;157:84-9.

10. Akkanti B, Hussain R, Nathan S, Gentry B, Young AY, Raissi F, Nascimbene A, Rajapreyar IN, Banjac ID, Patel K, Janowiak L, Patel M, Patel JA, Loyalka P, Gregoric ID, Kar B. Prolonged venovenous extracorporeal membrane oxygenation in a patient with acute respiratory distress syndrome. ASAIO J. 2016;62:e13-4.

11. Otterspoor LC, Smit FH, van Laar TJ, Kesecioglu J, van Dijk D. Prolonged use of extracorporeal membrane oxygenation combined with prone positioning in patients with acute respiratory distress syndrome and invasive aspergillosis. Perfusion. 2012;27:335-7.

12. Fan E, Gattinoni L, Combes A, Schmidt M, Peek G, Brodie D, Muller T, Morelli A, Ranieri VM, Pesenti A, Brochard L, Hodgson C, Van Kiersbilck C, Roch A, Quintel M, Papazian L. Venovenous extracorporeal membrane oxygenation for acute respiratory failure. Intensive Care Med. 2016;42:712-24.

13. Hough CL. Should we ever give steroids to ARDS patients? Clin Chest Med. 2014;35:781-95.

14. Sumi Y, Ogura H, Akashi K, Tohma Y, Tabuse H, Mizushima Y, Yokota J, Sugimoto H, Fujii C. Improved oxygenation by steroid pulse therapy in early-phase acute respiratory distress syndrome. JJAAM. 2007;18:1-9. 